\title{
EL NUEVO ESCENARIO DE LA REGULACIÓN DE LA ESTIBA Y DESESTIBA PORTUARIA EN EL ORDENAMIENTO JURÍDICO ESPAÑOL
}

\author{
Cristina Menéndez de la CRuZ \\ Funcionaria de Carrera de Habilitación Nacional \\ Doctora en Derecho Administrativo \\ Facultad de Derecho. Universidad de Valencia \\ cristina.menendez@uv.es
}

\section{ANTECEDENTES DEL MODELO DE ESTIBA Y DESESTIBA EN ESPAÑA}

A lo largo de este año 2017 ha ocurrido con la estiba lo que suele suceder cuando comienza una película y sólo con los primeros acordes de la banda sonora uno se aventura a decir: «esto huele a tragedia». Desde hace años, era de sobra conocido por todos los agentes implicados (autoridades portuarias, ejecutivos de diverso signo político, empresas estibadoras y trabajadores del sector) que el modelo español de estiba y desestiba portuaria contravenía las reglas del Derecho comunitario. En realidad, no ha habido nunca una verdadera intención de acometer una reforma del sector hasta que la película ha dado un giro. Las instituciones comunitarias se han encargado de que los personajes asuman cada uno su papel, obligando a acometer cambios en el modelo de funcionamiento del servicio de estiba y desestiba.

Los acontecimientos de los últimos meses han sido de sobra retransmitidos, han ocupado portadas de periódicos, noticias en televisión, han aparecidos tertulianos documentados y se ha dado hasta un esperpéntico ejercicio de política parlamentaria con un primer intento fallido de reforma que no pasó el trámite de convalidación en el Congreso mediante un Real Decreto-Ley 4/2017, de 24 de febrero, por el que se modifica el régimen de los trabajadores para la prestación del servicio portuario de manipulación de mercancías, dando cumplimiento a la Sentencia del Tribunal de Justicia de la Unión Europea (TSJUE) de 11 de diciembre de 2014, recaída en el asunto C-576/13 (procedimiento de infracción 2009/4052), publi- 
cado en el BOE, núm. 48, de 25 de febrero de 2017. En concreto, la Resolución de 16 de marzo de 2017, del Congreso de los Diputados, ordenó la publicación del Acuerdo de derogación de la norma.

El nuevo modelo de estiba y desestiba portuaria en España, después de varios avatares, y un nuevo trámite parlamentario, se establece en el Real Decreto-Ley 8/2017, de 12 de mayo, por el que se modifica el régimen de los trabajadores para la prestación del servicio portuario de manipulación de mercancías, dando cumplimiento a la Sentencia del TSJUE de 11 de diciembre de 2014. La primera cuestión relevante, y que se aprecia con una simple lectura del enunciado del nuevo texto legal, es el hecho de acudir a un real decreto-ley — que tal y como reconoce la CE de 1978 es una técnica pensada para supuestos de urgencia- para acometer una reforma que trae causa de una sentencia fechada en 2014 y de un procedimiento de infracción que se inicia en 2009. Pero llegados a este punto considero oportuno hacer primero un repaso de todas las vicisitudes que han rodeado a la estiba y desestiba portuaria en el ordenamiento jurídico español en los últimos años y que han dado lugar a la situación actual.

\section{LA SIGNIFICACIÓN ECONÓMICA DE LA ESTIBA Y DESESTIBA PORTUARIA Y SU EVOLUCIÓN CONCEPTUAL EN EL ORDENAMIENTO JURÍDICO ESPAÑOL}

Desde siempre se ha evidenciado la importancia de unos puertos bien gestionados con capacidad para ser motores de la economía. Los servicios que se acometen en el entorno portuario son básicos y funcionan como una suerte de muñecas rusas, unos encajan con los otros hasta conformar un correcto resultado; dentro de todo este proceso, la estiba y desestiba suponen un entramado de actividades relacionadas con la carga y descarga de los buques que operan en un determinado puerto. La actividad tiene una particularidad: la intermitencia en la demanda; esta situación es un hándicap que condiciona la regulación del propio servicio. Las necesidades de las labores de estiba y desestiba están subordinadas al número de buques que decidan operar en un puerto, circunstancia que no tiene una periodicidad fija, lo que da lugar a la imposibilidad de realizar una previsión de las necesidades de mano de obra a priori por medio de los llamados trabajadores estibadores. 
Para asegurar la correcta prestación del servicio se ha hecho necesaria históricamente la implicación de los poderes públicos. Se pueden resumir en tres los motivos que han propiciado la intervención pública en las actividades portuarias de estiba y desestiba:

a) El control sobre la profesionalidad de los trabajadores que realizan las actividades de estiba y desestiba, garantizando que siempre hubiera personal disponible para que los buques pudieran operar en puerto.

b) La garantía del correcto funcionamiento del puerto asegurando el abastecimiento de mercancías y el tránsito de las operaciones mercantiles que conforman las exportaciones e importaciones de un país.

c) El control sobre un espacio limitado como es el dominio portuario en el que se desarrolla el servicio de estiba y desestiba, delimitando los prestadores que pueden operar en un puerto.

El papel adoptado por el Estado ha ido variando desde un intervencionismo que llevó a la prestación directa del servicio, hasta mecanismos que pasan por la mera regulación en un contexto en el que la iniciativa privada es la encargada de la encomienda.

Analizando un plano más estrictamente jurídico, el servicio ha sufrido varias transformaciones, apreciándose una clara evolución de la condición de servicio público hacia una actividad económica de interés general en el servicio de estiba y desestiba portuaria. El carácter de servicio público en la estiba y desestiba portuaria se justifica por las notas de esencialidad, continuidad y calidad que requieren estas labores portuarias. La propia significación del servicio en el conjunto de la economía justifica la intervención de los poderes públicos con la finalidad de garantizar necesidades colectivas de interés general. El punto de partida ha sido una declaración como tal por medio de la denominada «publicatio»; con el paso del tiempo y la evolución de nuevas técnicas jurídicas han ido desapareciendo los incipientes monopolios públicos de intervención estatal dando paso a una liberalización de los servicios.

La incorporación de la iniciativa privada a la ejecución de actividades reservadas tradicionalmente a los Estados se debe en gran medida a los avances tecnológicos, aunque también a razones institucionales, políticas e ideológicas, sobre todo por exigencias del Derecho de la Unión Europea; transformaciones que lógicamente se han evidenciado en la estiba y desestiba portuaria. 
Los Estados sometidos a férreos controles del gasto público no han podido acometer grandes inversiones para adaptarse a los nuevos tiempos, así pues, se han ido ideando mecanismos para hacer confluir las necesidades públicas y la iniciativa privada. De este modo las técnicas concesionales han dado paso a las autorizaciones, los contratos de colaboración público-privada, etc. La actuación de los poderes públicos pasa de ser prestadores directos a controladores de la prestación, y la ejecución queda encomendada, en el nuevo escenario, a los agentes privados. Los poderes públicos reconducen sus cometidos a los de control de acceso a la prestación, limitación del número de prestadores sobre la base de una mejor utilización de los espacios públicos (evidente en el caso del dominio público portuario), la garantía de normas medioambientales, etc.

En ese nuevo modelo el Estado deja de ser prestador directo de servicios, pero se convierte en un estratega de excepción para garantizar que los tradicionales servicios públicos se sigan prestando bajo unas condiciones de continuidad y calidad. Los poderes públicos no sólo deben preservar las normas sobre competencia en un contexto de libre mercado, también existen otros derechos dignos de protección que afectan directamente a la esfera de los ciudadanos como usuarios directos o indirectos de los servicios. En el caso concreto del servicio de estiba y desestiba, la competencia de los poderes públicos no vincula sólo a los operadores portuarios, ya que, como se ha mencionado anteriormente, la trascendencia del servicio traspasa el entorno portuario y afecta a todo el conjunto de la economía y de la población.

También es importante mencionar la protección de otros intereses con relevancia económica conexos al servicio de estiba y desestiba portuario. Es evidente el conglomerado de intereses que subyacen bajo este servicio portuario en aspectos diversos. En primer lugar, la correcta utilización del dominio público portuario ha sido una constante en las legislaciones españolas en materia portuaria. Se trata de un recurso limitado, y el número de prestadores de servicios debe establecerse por parte de las autoridades portuarias. Los puertos de interés general forman parte del dominio público marítimo-terrestre e integran el dominio público portuario estatal. Las empresas que desean acceder a una licencia para la prestación del servicio de estiba y desestiba saben de facto que acceden a un servicio limitado; no todas las licencias del servicio implican necesariamente una concesión de dominio público, y sólo algunos operadores cuentan con ambos títulos administrativos. En general, se tiene derecho al acceso para el desarrollo del servicio portuario y al estableci- 
miento de la maquinaria necesaria para la estiba y desestiba por medio de autorización.

De otro lado, es relevante el establecimiento de redes de transporte transeuropeas y a escala mundial. La idea es crear espacios para el desarrollo de un transporte marítimo sin fronteras, dadas las ventajas que aporta en detrimento de otros medios de transporte como el habitual realizado por carretera. Para potenciar la competitividad de los puertos europeos es fundamental contar principalmente con un servicio de estiba y desestiba ágil y profesional, puesto que del tiempo de estancia en puerto dependerá que un buque lo elija como escala para operar. En ese contexto es interesante un marco común sobre los recursos humanos encargados de la puesta en marcha de los servicios portuarios asegurando unas buenas condiciones de trabajo. Medidas como las recogidas en el ordenamiento jurídico español hasta el momento sobre el sector de la estiba y desestiba portuaria, consecuentemente, no van en la dirección de potenciar esa movilidad geográfica ni de hacer atractiva la incorporación de nuevos operadores al sector.

Otro de los aspectos conexos es la mejora de las infraestructuras portuarias; el elevado coste del mantenimiento de las instalaciones portuarias urge a la necesidad de buscar mecanismos de colaboración público-privada.

Por último, otro de los aspectos relacionados con el servicio de estiba y desestiba portuario es la formación de nuevo personal. La oferta de formación para nuevos estibadores hasta ahora ha estado reservada a las sociedades de gestión, circunstancia que generaba una limitación de la competencia, puesto que el acceso estaba por regla general muy restringido. De otro lado, una formación facilitada por centros privados no parece muy rentable, porque en todo caso se debería articular una preparación práctica en el propio entorno portuario, dado que se requiere el manejo de compleja maquinaria, que sería difícil reproducir por medio de simuladores.

Todas estas cuestiones dan una idea de la relevancia que un servicio como la estiba y desestiba portuaria tiene en el contexto de la economía. A juicio de esta autora, la significación del servicio ha supuesto una rémora para acometer cambios. El excesivo celo que se tenía a que la situación descontrolara ejes económicos de gran relevancia ha paralizado todos los intentos de cambiar el modelo de estiba en España. Pero al final la deriva de los acontecimientos no ha podido evitar la eclosión de los conflictos entre las partes implicadas y que trascendiera a la opinión pública la percepción generalizada de que todo era inevitable, cuando lo cierto es que se podían haber evitado el conflicto si hubiera habido una verdadera inten- 
ción de ajustar el modelo a los postulados comunitarios, tal y como venía advirtiendo la Unión Europea desde diversas instancias.

\section{BREVE EXPOSICIÓN DE LA EVOLUCIÓN LEGISLATIVA DEL MODELO DE ESTIBA Y DESESTIBA PORTUARIA EN ESPAÑA}

En sus orígenes, la regulación del servicio se centró principalmente en un ámbito laboral. Con el paso de los años y la relevancia del servicio, éste pasó a ser considerado como un servicio que debía ser objeto de intervención y control por parte del Estado en un enfoque mucho más amplio. De un lado, la relación que mantienen los servicios de estiba y desestiba con el dominio público marítimo de titularidad pública y, de otro, la conexión de los servicios con el interés general de preservar el funcionamiento del tráfico marítimo.

La necesidad de asegurar una regularidad en la prestación del servicio fue lo que llevó a finales de los años ochenta en España a una primera regulación. Con el Real Decreto-Ley 2/1986, de 23 de mayo, sobre el servicio público de estiba y desestiba de los buques, se produce una declaración expresa por la que la actividad de estiba y desestiba de buques en los puertos de interés general pasa a tener la consideración de un servicio público esencial de titularidad estatal. El servicio sería prestado por empresas estibadoras privadas, pero la materialización del trabajo debía articularse por medio de las denominadas Sociedades Estatales de Estiba y Desestiba (SEED), con participación mayoritariamente pública y que tenían encomendada la gestión del personal estibador. La participación de las empresas estibadoras, concesionarias de un servicio público, en las SEED se establecía como obligatoria.

Posteriormente, con la Ley 27/1992, de 24 de noviembre, de Puertos del Estado y de la Marina Mercante, a pesar de pretenderse una incipiente privatización de servicios portuarios, los servicios de estiba y desestiba siguieron teniendo una consideración distinta y continuaron catalogados como servicios públicos. No será hasta la aprobación de la Ley 48/2003, de 26 de noviembre, de Régimen Económico y de Prestación de Servicios de los Puertos de Interés General, cuando verdaderamente puede observarse un golpe de timón en el ordenamiento jurídico español respecto de la gestión portuaria, y, en especial, por lo que se refiere a la estiba y desestiba. 
Como primer paso se transformaron las SEED en Agrupaciones Portuarias de Interés Económico (APIE), lo que conllevó la separación de las autoridades portuarias de las sociedades de gestión de trabajadores. Otro de los cambios trascendentes se produjo con la introducción del concepto de autoprestación, mediante el cual el servicio en algunos casos podía prestarse por personal del propio buque. Sin embargo, el cambio más relevante, y de ahí la denominada despublicatio, es que se abandona la concepción de servicio público para referirse a las labores de estiba y desestiba como servicios de interés económico general; en concreto, la ley los denomina servicios básicos. Con esta nueva regulación los servicios pasan a estar sujetos a las obligaciones de servicio público, obligaciones que se desarrollan en los pliegos reguladores del servicio, con la finalidad de garantizar su prestación en condiciones de seguridad, continuidad y regularidad, cobertura, calidad y precio razonable, sin olvidar el respeto al medio ambiente. Es evidente que de este modo se produce una sustitución de la tradicional técnica del servicio público por otro mecanismo de intervención más propio de los sectores liberalizados, como es el establecimiento de obligaciones de servicio público y así garantizar el interés general que persiste en la actividad a pesar de su liberalización.

Las ulteriores reformas hasta llegar al parcialmente derogado con la nueva regulación TRLPMM (Real Decreto Legislativo 2/2011, de 5 de septiembre) no han supuesto cambios sustanciales. La Ley 33/2010, de 5 de agosto, de modificación de la Ley 48/2003, de 26 de noviembre, de Régimen Económico y de Prestación de Servicios en los Puertos de Interés General, sólo supuso que las sociedades de gestión cambiaran a una nueva denominación como Sociedades de Gestión de Estibadores Portuarios (SAGEP). Con esta nueva figura, con participación exclusivamente privada, las sociedades de gestión estaban constituidas por las empresas titulares de licencias, con una distribución accionarial alícuota por el número de titulares y proporcional por el volumen de facturación. A mi juicio, este cambio de denominación no es más que un nuevo nombre, pero en el fondo el modelo continuó inmutable.

Éste es el marco legal que viene a derogar o modificar el Real DecretoLey 8/2017, de 12 de mayo, en su disposición derogatoria única y disposición final primera. Pero antes de entrar en el análisis del nuevo modelo es interesante valorar los porqués que han propiciado la intervención de las instituciones comunitarias. El proceso ha sido largo, se ha hablado mucho en los medios de comunicación sobre la cuantiosa multa que pesa sobre el 
Reino de España por no atender a los mandatos comunitarios, pero se ha obviado decir que las instituciones comunitarias dieron muchísimos avisos previos antes de acabar imponiendo el TSJUE una sanción económica; advertencias que nunca fueron atendidas y contra las que incluso el gobierno español se opuso de manera vehemente.

En este sentido es sumamente interesante la respuesta del Ministerio de Fomento español de 27 de diciembre en contestación al Dictamen motivado de infracción núm. 2009/4052, de fecha 1 de octubre de 2012, emitido desde las instituciones comunitarias, como paso previo a un proceso de sanción. En aquel momento el ejecutivo español exponía con fervor en sus líneas argumentales que las obligaciones impuestas por la ley española para el acceso a la prestación del servicio de estiba y desestiba portuaria se justificaban por razones imperiosas de interés general.

Las autoridades españolas recurrieron entonces al argumento de las obligaciones de servicio público como cargas o deberes que se imponían a las empresas privadas prestadoras de servicios, que de otro modo no asumirían si sólo atendieran a su beneficio empresarial, pero que el poder público argumentaba debía imponer en aras a la consecución de objetivos de interés general. Estas obligaciones, que en otros sectores son claras, a juicio de esta autora siempre se ha mantenido que era más que cuestionable que se pudieran extrapolar, sin matices, al sector de la estiba. Pues bien, esos planteamientos, que ahora parecen haberse evaporado, son los que llevaron a una inactividad que trajo como consecuencia la sanción de la Unión Europa.

\section{CAUSAS QUE HAN PROPICIADO LA INTERVENCIÓN DE LAS INSTITUCIONES COMUNITARIAS: INCORRECTA INCORPORACIÓN DE OBLIGACIONES DE SERVICIO PÚBLICO EN UN CONTEXTO DE LIBERALIZACIÓN}

\section{Un modelo que ha restringido la libre concurrencia}

El Derecho interno no ha sabido en todos estos años adaptar las normas de Derecho comunitario relativas al establecimiento de obligaciones de servicio público, pues tras una aparente intencionalidad de cumplir un mandato público de asegurar la prestación de un servicio público se ha construido un modelo de estiba que constriñe la incorporación de nuevos operadores. 
Las empresas estibadoras que han querido operar en España se han topado con la imposición de unas supuestas obligaciones de servicio público que no se corresponden con las exigencias de continuidad y regularidad del servicio de estiba y desestiba:

a) Para obtener una licencia que permita operar en un puerto de interés general se exige a las empresas estibadoras participar financieramente en el capital de una sociedad de gestión de trabajadores portuarios.

b) Otro requisito imprescindible para el acceso a la licencia consistía en cumplir con una serie de imposiciones de tipo laboral que afectan a su propia planificación empresarial, como es el hecho de contratar con carácter prioritario a un determinado número de trabajadores de forma indefinida. Esta contratación, además, no podía hacerse en el mercado libre, sino que tenía que tratarse de trabajadores inscritos previamente en la sociedad de gestión.

La justificación a las restricciones a la libertad de empresa deben ampararse siempre en razones imperiosas de interés general, razones que sí se dan en el caso concreto del servicio portuario de estiba y desestiba: de un lado, la protección de los trabajadores y, de otro, garantizar la regularidad, continuidad y calidad del servicio portuario. La forma de articular esas restricciones por medio de las denominadas obligaciones de servicio se ha efectuado de modo incorrecto; hubieran debido articularse mecanismos más proporcionados al fin perseguido y que respondieran igualmente a la finalidad perseguida.

\section{Una errónea transposición de las reglas de Derecho comunitario}

Con la Sentencia del TSJUE de 11 de diciembre de 2014 culmina un proceso instado desde las instancias comunitarias por el que se ha puesto en cuestión el modelo de estiba y que puede resumirse en un incumplimiento de las reglas de la competencia.

La relación del servicio de estiba y desestiba portuario en España con el Derecho comunitario ha sido un tanto convulsa; ha pasado de ser un modelo que acoge los postulados comunitarios, cuando ni siquiera las instancias comunitarias llegan a materializarlos, tal y como ocurrió con la regulación de la Ley del año 2003, que se aventuró a recoger postulados 
de una Propuesta de Directiva de 2001 sobre la estiba portuaria en Europa que no llegó a materializarse, a posteriormente ser objeto de condena porque el modelo contraviene los postulados comunitarios.

La UE ha pretendido desde hace muchos años una armonización de la regulación de todo el transporte marítimo comunitario no sólo por la relevancia de los intercambios intracomunitarios, sino también por la relevancia de Europa, dada su posición estratégica a escala mundial. En las instancias comunitarias ha sido difícil consensuar la técnica del servicio público, puesto que la tradición jurídica de los diferentes Estados aportaba ideas muy diversas, pero sí se ha llegado a un planteamiento común: el Derecho comunitario no aboga por la abolición de la técnica del servicio público, sino que éste debe ser adecuado a un contexto de libre competencia. Para ello se ha optado por nuevos mecanismos que afectan a la organización y régimen jurídico de los tradicionales servicios en Europa.

No basta con que las normas legales recojan en su texto que el servicio se liberaliza. Esta declaración de intención debe materializarse y, en el caso de la estiba y desestiba portuaria en España, no se ha producido correctamente. Las restricciones a la libertad de establecimiento de nuevos operadores constituyen un obstáculo que va más allá de lo que resulta necesario para alcanzar los objetivos perseguidos, y, por tanto, no parece correcta la transposición de la normativa comunitaria. La idea subyacente de esta mala praxis es soterrar un proteccionismo del Estado en detrimento de la libertad de establecimiento, un hecho que no puede justificarse desde la UE donde preconizan las reglas del libre mercado.

\section{LA PRETENDIDA LIBERALIZACIÓN DEL SECTOR DE LA ESTIBA Y DESESTIBA PORTUARIA EN EL NUEVO MARCO LEGAL DEL REAL DECRETO-LEY 8/2017, DE 12 DE MAYO. ASPECTOS MÁS RELEVANTES DE LA NUEVA NORMA}

El Real Decreto-Ley 8/2017, de 12 de mayo, tiene como finalidad establecer el régimen de los trabajadores para la prestación del servicio portuario de manipulación de mercancías (los estibadores), adaptando el Derecho interno para que resulte compatible con la libertad de establecimiento consagrada en el art. 49 del Tratado de Funcionamiento de la Unión Europea. 
El texto legal entró en vigor el 14 de mayo de 2017, al día siguiente de su publicación en el Boletín Oficial del Estado ${ }^{1}$. No obstante, algunas disposiciones gozan de un régimen transitorio, tal y como se establece para el apartado 4 de la disposición transitoria primera, relativo a las Sociedades Anónimas de Gestión de Estibadores Portuarios (SAGEP), que no entrará en vigor hasta la expresa declaración por la Comisión Europea de su compatibilidad con el régimen de ayudas de Estado.

Mención distinta merece el hecho de que la entrada en vigor del nuevo marco legal ha venido acompañada de una férrea oposición por parte de los trabajadores, llegando a producirse jornadas de huelga, pero finalmente se ha alcanzado un acuerdo a finales de junio de 2017 entre los estibadores portuarios y algunas de las empresas del sector. En líneas generales, el principio de acuerdo recoge cuestiones positivas para el sector en relación con las medidas organizativas y productivas, así como respecto a las garantías en la continuidad en el empleo de los actuales trabajadores.

Por lo que se refiere a la estructura de la norma, se compone de cuatro artículos. El primer artículo define el objeto, quedando éste referido al cumplimiento de la Sentencia de 11 de diciembre de 2014 del TSJUE.

Un segundo artículo parece más bien una aseveración del legislador para enfatizar sus intenciones, al afirmar en un artículo una cuestión más propia de las exposiciones de motivos: «La libertad de contratación de trabajadores portuarios para la prestación del servicio portuario de manipulación de mercancías». El artículo en sus dos apartados recalca que la contratación será libre y que además las empresas estibadoras para la prestación del servicio de manipulación de mercancías sólo necesitarán obtener la pertinente licencia, no estando obligadas, como hasta ahora, a participar en ninguna empresa cuyo objeto social sea la puesta a disposición de trabajadores portuarios.

El tercer artículo alude a cuestiones sobre la capacitación de los trabajadores estibadores, materia sobre la que me detendré más adelante al analizar mi visión global de la nueva configuración de la estiba portuaria en España y examinar con más detalle uno de los grandes temas olvidados en esta norma: la formación y acceso de nuevos trabajadores.

El último artículo, el cuarto, regula los denominados Centros Portuarios de Empleo. Al respecto es importante matizar que la sentencia del TSJUE que trae causa al Real Decreto-Ley 8/2017 no predeterminaba la fórmula legalmente aplicable para adaptar el sector de la estiba y desestiba

\footnotetext{
${ }^{1}$ BOE, núm. 114, de 13 de mayo de 2017.
} 
portuaria en España. El fallo judicial señalaba en concreto: «Las restricciones inherentes al régimen portuario español no son adecuadas ni necesarias para alcanzar el objetivo de garantizar la regularidad, continuidad y calidad del servicio, ya que tal objetivo podría conseguirse con medidas menos restrictivas, a semejanza de las adoptadas en otros Estados miembros, tales como la gestión por las propias empresas estibadoras de oficinas de empleo para disponer de mano de obra, de modo que dichas empresas puedan contratar libremente trabajadores permanentes o temporales, o la creación de una reserva de trabajadores gestionada por empresas privadas, que funcionen como agencias de empleo temporal y que pongan trabajadores a disposición de las empresas estibadoras».

Así pues, se pueden extraer como admisibles dos opciones:

- Bien que fueran las propias empresas estibadoras las que, pudiendo contratar libremente trabajadores permanentes o temporales, gestionaran unas oficinas de empleo que les facilitarían mano de obra y además impartieran la formación de los trabajadores.

- Otra alternativa era la creación de una reserva de trabajadores gestionada por empresas privadas que funcionen como agencias de empleo temporal y que pongan trabajadores a disposición de las empresas estibadoras.

Como se ha mencionado, el nuevo marco legal establece en el art. 4 del Real Decreto-Ley 8/2017 la creación de Centros Portuarios de Empleo, optando por una completa liberalización, puesto que permite que la instauración de estos centros pueda realizarse por cualquier persona natural o jurídica, o uniones y entidades sin personalidad jurídica, no cercenando la creación únicamente a las empresas estibadoras que operen en puertos españoles. En resumen, estos centros operarán como empresas de trabajo temporal específicas para el sector, cumpliendo en todo momento con la Ley 14/1994, de 1 de junio, que regula las empresas de trabajo temporal, cuya literalidad les será de plena aplicación.

Siguiendo con el análisis de la estructura del texto legal, el Real Decreto-Ley 8/2017 se preocupa más de determinar un régimen transitorio que de crear un nuevo orden normativo efectivo que constituya verdaderamente un nuevo modelo de estiba y desestiba en los puertos españoles.

La norma prevé un proceso transitorio de tres años que permita un tránsito ordenado al nuevo régimen de libertad de contratación en la disposición transitoria primera. Durante el periodo transitorio podrán subsistir las SAGEP, pero una vez concluido el periodo transitorio, las SAGEP 
podrán continuar desarrollando su actividad en régimen de libre competencia, siempre que cumplan los requisitos establecidos con carácter general para las empresas de trabajo temporal.

En el plazo máximo de los primeros seis meses del periodo, los accionistas de las SAGEP deberán decidir individualmente si desean continuar o separarse de las mismas, en cuyo caso sus acciones serán adquiridas por los accionistas que permanezcan o, a falta de ellos, serán amortizadas con la consiguiente reducción de capital. Si ningún accionista quisiera permanecer en las SAGEP, éstas se disolverá de acuerdo con las reglas generales establecidas en la Ley de Sociedades de Capital.

Otra cuestión peliaguda de la nueva norma es el tema de la extinción de los contratos de los trabajadores de las SAGEP y la asunción de las indemnizaciones en caso de rescisión del contrato laboral por parte de las autoridades portuarias. Con el objeto de igualar la posición competitiva entre los actuales titulares de licencias de prestación del servicio portuario de manipulación de mercancías con aquellos nuevos que accedan a la prestación, en el caso de que en cualquier momento posterior a la entrada en vigor del real decreto-ley se produjera la extinción de contratos laborales de los trabajadores de las SAGEP vigentes antes del 11 de diciembre de 2014, o de los suscritos en régimen laboral común por las empresas titulares de licencia con trabajadores procedentes de las SAGEP por imperativo legal con anterioridad a dicha fecha, por cualquiera de las causas contempladas en los arts. 51 y 52 del Estatuto de los Trabajadores de 2015, la autoridad portuaria correspondiente al ámbito geográfico de las SAGEP donde dichos trabajadores estuvieron contratados asumirá obligatoriamente el 100 por 100 de las indemnizaciones procedentes por esta causa en la cuantía prevista en el art. 53 del ET 2015, generadas desde la celebración de los contratos hasta su extinción o hasta la finalización del periodo transitorio si fuera anterior.

No obstante, esta disposición no entrará en vigor hasta la expresa declaración por la Comisión Europea de su compatibilidad con el régimen de ayudas de Estado, tal y como se ha mencionado antes al hablar de la entrada en vigor del Real Decreto-Ley 8/2017.

Dado que la visión de esta autora se centra sobre los aspectos de relevancia jurídica desde el punto de vista de la regulación del servicio, en este artículo no me voy a detener en un análisis pormenorizado de las cuestiones estrictamente laborales, dado que éstas deberán ser objeto de la pertinente negociación colectiva, como así además lo establece la disposición adicional primera del Real Decreto-Ley 8/2017 al señalar que en el plazo 
máximo de un año desde la entrada en vigor del texto legal las normas convencionales vigentes deberán adaptarse a lo dispuesto en el mismo. Igualmente, el cese de las movilizaciones por parte de los trabajadores se ha debido al acuerdo alcanzado con algunas de las empresas estibadoras con más implantación en los puertos españoles de regularizar la situación atendiendo a algunas de las reivindicaciones de los trabajadores. En particular, se recoge en el acuerdo alcanzado en junio de 2017 la garantía de mantener el 100 por 100 del empleo de los actuales trabajadores que desempeñan labores de estiba y desestiba en los puertos españoles, implementando igualmente sistemas de prejubilaciones o bajas voluntarias incentivadas.

\section{REFLEXIÓN FINAL Y ANÁLISIS SOBRE EL INCIERTO FUTURO DEL MODELO DE ESTIBA Y DESESTIBA EN ESPAÑA}

La sentencia condenatoria del TSJUE ha sido un punto de inflexión que ha abocado a los agentes intervinientes al consenso final, pero antes se ha dado un espectáculo poco edificante. Algunos de los comportamientos que se han visto por todas las partes son propios de una tragedia de Shakespeare, como aludía al principio de este artículo.

Los problemas que acucian al sector de la estiba y desestiba portuaria en España sólo podrán solventarse por medio de una nueva y profunda regulación; el éxito dependerá no sólo de las decisiones que se adopten, sino de cómo estas se pongan en práctica. A mi juicio, el Real Decreto-Ley 8/2017 no aporta una verdadera regulación del servicio; parchea, tarde $-\mathrm{y}$ no con las mejores formas-, una situación insostenible de incumplimiento de los mandatos comunitarios.

La regulación de la actividad exige, en mi criterio, un cambio en el modelo, en particular atendiendo a lo siguiente:

1. Un replanteamiento del papel que han jugado hasta el momento las autoridades portuarias. La iniciativa privada debe tener presente que, si bien puede participar en la economía mediante la prestación de servicios con trascendencia para la colectividad, no puede olvidar que está sujeta a la supervisión de las autoridades públicas. Por mi parte abogo por dotar de mayor relevancia a los mecanismos de inspección con los que cuentan los poderes públicos, sobre todo desde el ámbito sancionador; potestad que es inherente al poder público y sobre la que puede construirse un dispositivo de control fundamental para la efectividad de los servicios públi- 
cos. No se trata de imponer un régimen sancionador con finalidad recaudatoria, sino que induzca al cumplimiento de normas básicas para el buen funcionamiento, en este caso del entorno portuario, en materia laboral, medioambiental, defensa de los consumidores, etc. Cuestiones sobre las que en ningún caso se ha aludido en la nueva norma y sobre las que únicamente se modifican algunos artículos del texto refundido de la Ley de Puertos del Estado y de la Marina Mercante (TRLPMM) en la disposición final primera del Real Decreto-Ley 8/2017, sin aportar una verdadera nueva regulación del acceso al servicio de manipulación de mercancías.

2. La actividad de estiba y desestiba portuaria no puede ser una actividad totalmente liberalizada, debe establecerse un modelo que pase necesariamente por la previa autorización de las autoridades portuarias. En primer lugar, para regular el uso de un recurso limitado como es el dominio público portuario y, en segundo lugar, para asegurar que las empresas operadoras lo hacen bajo unos determinados estándares de calidad y seguridad. Este ámbito debe presuponerse que se deja a la libertad reguladora de los pliegos que las autoridades portuarias realicen para acceder a la prestación del servicio. Pero no parece muy congruente con la postura mantenida hasta el momento por el legislador español sobre la importancia de mantener unas obligaciones de servicio público en el servicio de estiba y desestiba portuaria.

3. En algunos ámbitos concretos debe potenciarse la autoasistencia. No tiene ninguna justificación que labores de estiba y desestiba portuaria que no requieran de una gran especialización no puedan ejecutarse por personal de los propios buques. Al respecto se establece en la disposición final primera del Real Decreto-Ley 8/2017 una nueva redacción del art. 133.1 TRFLPMM $^{2}$, pero no se determina una reformulación de esta

\footnotetext{
${ }^{2}$ El apartado 1 del art. 133 tendrá la siguiente redacción: «A los efectos de esta ley se considera autoprestación cuando el concesionario o el titular de una terminal de pasajeros o de mercancías dedicada al uso particular se presta a sí mismo los servicios al pasaje o de manipulación de mercancías, respectivamente. También se considerará autoprestación cuando una compañía naviera se presta a sí misma uno o varios servicios portuarios con personal propio embarcado para los servicios a bordo y material propio, sin que se celebre ningún tipo de contrato con terceros a efectos de tal prestación. En el caso de servicios de transporte marítimo de corta distancia regulares y de las autopistas del mar realizados con buques ro-ro puros, ro-pax, con-ro y ferries se podrán prestar a sí mismo los servicios al pasaje y de manipulación de mercancías utilizando también personal propio en tierra. En ningún caso se podrá autorizar para aquellos buques que enarbolen el pabellón de un Estado incluido en la lista negra que se publica en el informe anual del Memorándum de París o, independientemente de su pabellón, que estén considerados como de alto o muy alto riesgo por el nuevo régimen de inspección».
} 
técnica que, en mi opinión, se ajustaría más al funcionamiento de otros puertos en el contexto internacional, donde la autoprestación es una fórmula bastante utilizada.

4. El mecanismo más apropiado para la efectiva ejecución del servicio de estiba y desestiba portuaria es ciertamente la creación de Centros Portuarios de Empleo, puesto que aseguran una gran bolsa de empleo disponible en todo momento para ejecutar la actividad. La creación de estos centros nunca debe ser una imposición de las autoridades públicas que altere la propia configuración empresarial de las operadoras que licitan y optan a prestar un servicio; ésta debe ser una decisión libre a iniciativa de las empresas estibadoras que decidan su constitución. Con la creación de estos Centros Portuarios de Empleo se sustentan los costes laborales de forma conjunta, un modelo muy similar al de las empresas de trabajo temporal. Pero aunque el nuevo marco legal establece su creación, creo que por la vía reglamentaria deberá establecerse correctamente su funcionamiento, porque si bien deben ajustarse a lo establecido para las empresas de trabajo temporal comunes, las especificidades del entorno portuario deben tenerse en cuenta y establecer una regulación pormenorizada (por ejemplo, para los supuestos de falta de mano de obra cualificada).

5. Las condiciones laborales de los estibadores deben estar garantizadas a pesar de la estacionalidad del trabajo. Para lograr este loable objetivo no es necesario optar por medidas restrictivas, como ha ocurrido hasta ahora, de la propia libertad empresarial de los operadores del servicio. Igualmente, debe potenciarse la movilidad geográfica de trabajadores; empresas estibadoras extranjeras que opten a operar en puertos españoles no tendrían por qué encontrarse con problemas para contratar trabajadores de otros países, siempre que éstos cumplan con los requisitos de cualificación requeridos. El control sobre la profesionalidad de los trabajadores (nacionales o extranjeros) debe ser una de las encomiendas de las autoridades portuarias. $\mathrm{Al}$ respecto, y de nuevo recalcando que no es mi intención detenerme en cuestiones de estricto ámbito laboral, sí creo que una completa regulación del servicio de estiba y desestiba debe ocuparse con más detalle de la capacitación de los trabajadores y de sus condiciones laborales. La escueta regulación del art. 3 del Real Decreto-Ley 8/2017 sobre los requisitos de capacitación se queda escasa, pues alude únicamente a la obtención del certificado de profesionalidad para poder acceder a la profesión de estibador.

6. Por último, una mención a uno de los aspectos que más relevancia creo que tiene en la configuración de un nuevo modelo de estiba y des- 
estiba portuaria, y es que en materia de formación las autoridades portuarias pueden y deben jugar un papel relevante. Aunque el modelo siga sustentándose en la creación de centros de empleo al estilo de las empresas de trabajo temporal, éstos, a mi juicio, no tienen por qué ser los encargados de la formación y selección de nuevos trabajadores; esta encomienda puede dejarse en manos de las autoridades portuarias, que pueden acometer esta función con total imparcialidad. Mientras no se acometa una nueva regulación de estos aspectos, no estará garantizada la completa liberalización del servicio, puesto que no se garantiza que nuevos trabajadores puedan acceder al mercado laboral de la estiba y desestiba, en tanto que no se ha instaurado un marco para la formación de nuevos estibadores.

Como conclusión final el nuevo rol de los Estados debe ir encaminado hacía la garantía de la prestación de los servicios; ya no son sujetos de acción material que ejecutan directamente los servicios, sino que ahora el Estado pasa a adoptar un papel de coordinador de un proceso mucho más amplio. En el caso de la estiba y desestiba portuaria, si bien las leyes sí han recogido una liberalización del servicio, pasando éste a manos privadas, las autoridades portuarias no han sabido adaptarse a su nuevo cometido. La nueva regulación que se ha acometido en España es fruto de la desidia, de las prisas y, sobre todo, del toque de queda que impuso la Unión Europea con la imposición de multas tras la pasividad de los agentes intervinientes en el servicio de estiba y desestiba portuaria. No creo, sinceramente, que esta nueva regulación sea la forma global y exhaustiva que necesita el servicio para dinamizar el modelo sin que la actividad pierda sus notas características de servicio público. Sólo queda hacer un último acto de fe y confiar en que el desarrollo reglamentario cubra algunas de las inmensas lagunas que deja el nuevo marco legal de la estiba y desestiba portuaria en España, pero mi instinto no tanto jurídico, sino literario, me dice que tenemos tragedia shakesperiana al menos para un par de actos más. 